\title{
AMORPHOUS CARBON ACTIVE CONTACT LAYER FOR RELIABLE NANOELECTROMECHANICAL SWITCHES
}

Daniel Grogg ${ }^{1}$, Christopher L. Ayala', Ute Drechsler ${ }^{1}$, Abu Sebastian ${ }^{1}$, Wabe W. Koelmans ${ }^{1}$, Simon J. Bleiker ${ }^{2}$, Montserrat Fernandez-Bolanos ${ }^{1}$, Christoph Hagleitner ${ }^{1}$,

\author{
Michel Despont ${ }^{3}$ and Urs T. Duerig ${ }^{1}$ \\ ${ }^{1}$ IBM Research - Zurich, Switzerland \\ ${ }^{2}$ KTH Royal Institute of Technology, Sweden \\ ${ }^{3}$ IBM Research - Zurich, now at CSEM, Switzerland
}

\begin{abstract}
This paper reports an amorphous carbon (a-C) contact coating for ultra-low-power curved nanoelectromechanical (NEM) switches. a-C addresses important problems in miniaturization and low-power operation of mechanical relays: i) the surface energy is lower than that of metals, ii) active formation of highly localized a-C conducting filaments offers a way to form nanoscale contacts, and iii) high reliability is achieved through the excellent wear properties of a-C, demonstrated in this paper with more than 100 million hot switching cycles. Finally, a full inverter using a-C contacts is fabricated to demonstrate the viability of the concept.
\end{abstract}

\section{INTRODUCTION}

Mechanical switches, with their abrupt (on-off) $I-V$ characteristic offer, a distinct feature not found in other electronic devices. Recent research results highlight the importance of the contact characteristics for the operation of electromechanical relays. In particular, the switching energy of a NEM switch is limited by the size and the nature of its contact [1]. This means that to construct truly low-power NEM switches contact adhesion forces in the $\mathrm{nN}$ range must be achieved.

Miniaturization of the contact reduces the adhesion forces in a metallic contact [2]; however, the size of a single contact asperity and the tendency of metals to form a neck impose practical limits. Contacts using non-metallic materials, such as graphene [3], carbon nanotubes [4] and metal oxides $[5,6]$, have been explored as a means to achieve high reliability and low adhesion forces in NEM relays. Nonetheless, finding a suitable trade-off between good electrical contact and a low adhesion force remains a challenging task.

In this paper, we investigate a-C as contact material for NEM switches. The a-C used as a coating has a low surface energy, similar to other carbon-based materials, and it can be deposited with a simple physical sputter-deposition process suitable for full wafer integration. Moreover, Jouleheating-induced clustering of $\mathrm{sp}^{2}$ hybridized carbon atoms [7] enables current conduction in a localized filament without creating excessive adhesion force, thus enabling NEM relays to operate with a restoring force well below $100 \mathrm{nN}$.

\section{NEM SWITCH}

\section{Device Design}

The silicon NEM switch shown in Figure 1a is designed using the curved cantilever approach described in [8]. The motion of the free-standing cantilever can be described as a rotation around the hinge, which is close to the source. The hinge region is used to design the equivalent stiffness of the switch, which is $1.7 \mathrm{~N} / \mathrm{m}$ for this design. The contact of these devices is shown in detail in Figure $1 \mathrm{~b}$ and $1 \mathrm{c}$ in the open and closed state, respectively. In the open state, the actuation gap is about $60 \mathrm{~nm}$ wide, as is also the gap at the tip of the switch. When the tip comes into contact, the actuation gap remains open and is designed to remain half the width of the initial gap.
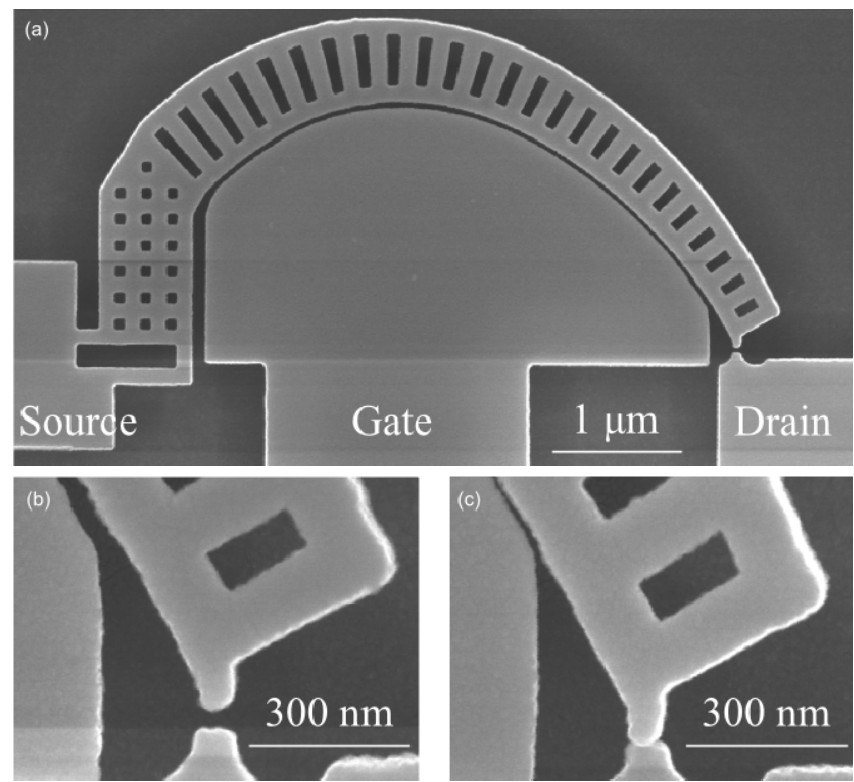

Figure 1: a) SEM micrograph of NEM switch fabricated. b) Contact region in the open and c) the closed state.

The effective benefit of the curved switch design is shown in Figure 2. The curvature helps avoid the excessive field strength often observed at the edge of a straight cantilever, which causes breakdown at low voltages. To confirm the advantages of the curved design experimentally, we fabricated both straight and curved cantilevers, as depicted in Figure 2a, on the same substrate next to each other. Figure $2 b$ reports the lowest and highest values for the pull-in and pull-out voltages as well as the breakdown 
voltages (destructive test) of three devices of each design. The curved and straight switches have similar pull-in and pull-out voltages, as expected from simulation. However, to confirm the influence of the curvature on these two characteristics a larger number of devices would be necessary. In contrast, the increase in breakdown voltage can be distinguished even on this small sample of devices and confirms a breakdown voltage of $>13.5 \mathrm{~V}$. This is more than twice the pull-in voltage in the worst case. Moreover, it clearly increases the margin between pull-in and breakdown compared with a straight cantilever.
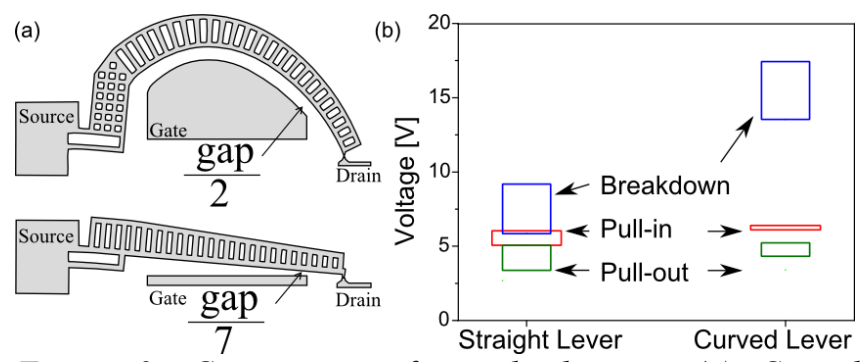

Figure 2: Comparison of switch designs: (a) Curved cantilever has a larger gap in the closed state, resulting in (b) higher measured breakdown voltages and smaller hysteresis windows.

\section{Fabrication}

The curved NEM switch shown in Figure 1 was fabricated using electron beam lithography on a silicon-oninsulator (SOI) substrate and the fabrication process described in Figure 3. The silicon device layer is $220 \mathrm{~nm}$ thick and etched using an ICP coupled HBr plasma to create vertical and smooth surfaces (step 1). The 60-nm air-gap is directly etched in the same process step, followed by sacrificial etching of the underlying silicon dioxide (step 2). A conduction layer is evaporated onto the device, enabling a low series resistance along the suspended cantilever of the switch (step 3). Good results have been achieved using platinum as conduction layer, but the inherent stress in the platinum layer creates a strong out-of-plane bending of the suspended parts of the NEMS switch. Therefore, a thin gold layer is used to relax the stress in the platinum layer. A low stress with nearly no visible out-of-plane bending is achieved using subsequent evaporation of the two materials while maintaining the harder platinum closer to the contact surface. Finally, a 10-nm-thin a-C layer is deposited over all devices using sputter deposition from a graphite target (step 4). The deposition is done in argon atmosphere, optimized for low stress in the as-deposited a-C layer. The main drawback of this process comes from potential electrical shortcuts created by the a-C layer. However, this drawback can be eliminated rather simply by using an additional photolithography step in the future.

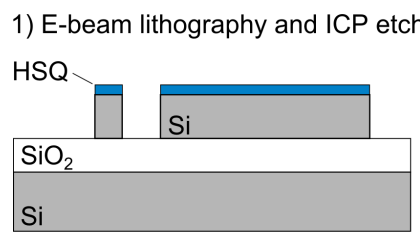

2) BHF release and super critical point drying

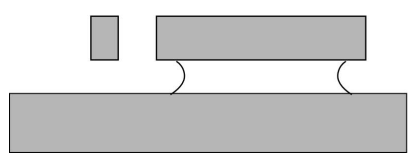

4) Amorphous carbon sputtering $X X X X X X$

Figure 3: NEM switch fabrication process with $a-C$ sputtering as the final step.

\section{CONTACT MATERIAL}

Static Characteristics

A schematic cross section through the contact region is shown in Figure 4a. The contact tip fabricated is $30 \mathrm{~nm}$ wide and has a slightly curved shape because of fabricationrelated effects. The resistivity of the as-deposited a-C carbon is high, and the measured $I-V$ characteristic fits a modified Poole-Frenkel model that accounts for the nonisolated nature of the traps arising from the high trap-density intrinsic to a-C (Figure $4 b)$.

A conduction filament forms in the a-C upon application of a sufficiently high voltage; currently voltages in the range of 1.5 to $2.5 \mathrm{~V}$ are necessary. This filament formation process is an active process, i.e., the filament is created post-fabrication during the first contact events. After this process, the filament remains in the a-C layer even when the switch is opened. Detailed investigations of the filament-formation process in $\mathrm{a}-\mathrm{C}$ at the nanoscale, including the field/temperature dependence, had been performed using a conductive-mode AFM [7]. Once the filament has been formed, there is a marked difference in the conduction behavior: metallic transport is observed, and the total resistance of the switch reduces to $15 \mathrm{k} \Omega$. This resistance depends on several factors, including the effective contact area, the layer thickness and the current flow (heat) during the formation process.

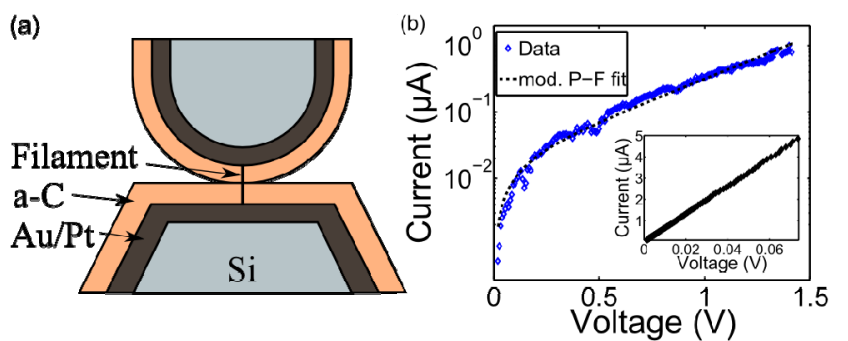

Figure 4: (a) Schematic cross section of contact region. (b) Experimental I-V characteristics of the drain-source contact after fabrication fit a modified Poole-Frenkel $(P-F)$ conduction model. Inset: Nearly perfect metallic conduction after formation of a filament in the $a-C$. 

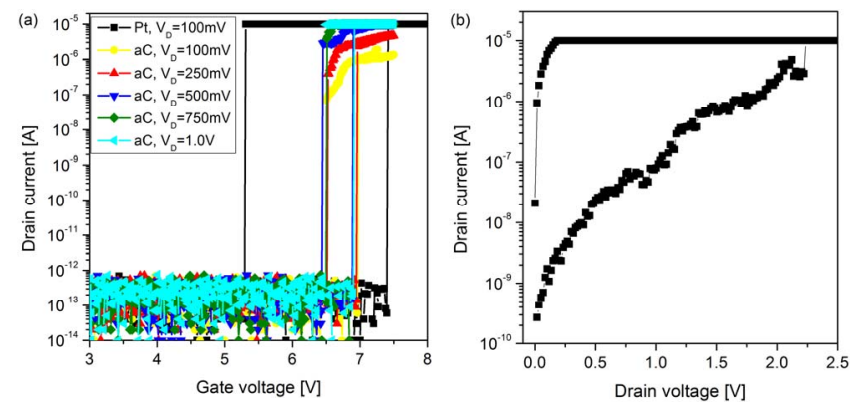

Figure 5: (a) $I_{D^{-}} V_{G}$ characteristic of a NEM relay: The larger hysteresis of the Pt contact (black) considerably reduces when using a-C coating. (b) $I_{D^{-}} V_{D}$ characteristic: Filament formation in a fresh a-C layer occurs at $2.2 \mathrm{~V}$.

The benefit of the a-C coating is evident in the $I_{\mathrm{D}}-V_{\mathrm{G}}$ characteristic (Figure 5a): the hysteresis window of the switch reduces from $>2 \mathrm{~V}$ for a platinum-platinum contact to $0.5 \mathrm{~V}$ for the a-C contact. Based on results from electromechanical simulations of the NEM switch, these hysteresis windows correspond to adhesion forces of about $50 \mathrm{nN}$ for the platinum contact and only $10 \mathrm{nN}$ for the a-C contact. Moreover, the resistance of the contact directly influences the maximum voltage the contact can handle during a measurement before stiction, neck formation and microwelding are observed. Using the a-C contact layer, static measurements with a current compliance of $10 \mu \mathrm{A}$ are possible up to $2.5 \mathrm{~V}$. Figure $5 \mathrm{~b}$ shows the $I_{\mathrm{D}}-V_{\mathrm{D}}$ characteristic of a fresh a-C layer with filament formation at $2.2 \mathrm{~V}$.

\section{Reliability}

Devices have been subjected to a long-term reliability test to assess the lifetime of nanoscale a-C contacts. The devices were operated using a gate voltage pulse of $-10 \mathrm{~V}$ at $50 \mathrm{kHz}$. The switching transient ramps have been $200 \mathrm{~ns}$ long, i.e., much slower than the simulated switching time of $\leq 42$ ns.

A two-level drain voltage is used to create a filament in the a-C layer at each contact cycle, if necessary, and to extract the total resistance at a lower voltage. The drain voltage levels are set to $V_{1}$ and $V_{1} / 10$, to extend the measurement range of the linear transimpedance amplifier used as an output. In addition, this voltage pulse also mimics the voltage conditions a NEM switch sees under capacitive load: a high voltage when closing the contact and a low voltage when opening the switch.

Figure 6 reports the extracted total ON-resistance $\left(R_{\mathrm{ON}}\right)$ for each switching cycle under hot switching conditions. One experiment was run with a drain voltage $V_{1}$ of $1.6 \mathrm{~V}$ $\left(0.16 \mathrm{~V}\right.$ for the extraction of $R_{\mathrm{ON}}$, plotted in red) and the other with $V_{1}=2.0 \mathrm{~V}\left(0.2 \mathrm{~V}\right.$ for the extraction of $R_{\mathrm{ON}}$, plotted in blue). These cycling tests with more than 100 million cycles at $1.6 \mathrm{~V}$ and $2.0 \mathrm{~V}$ prove the high reliability of the a-C.

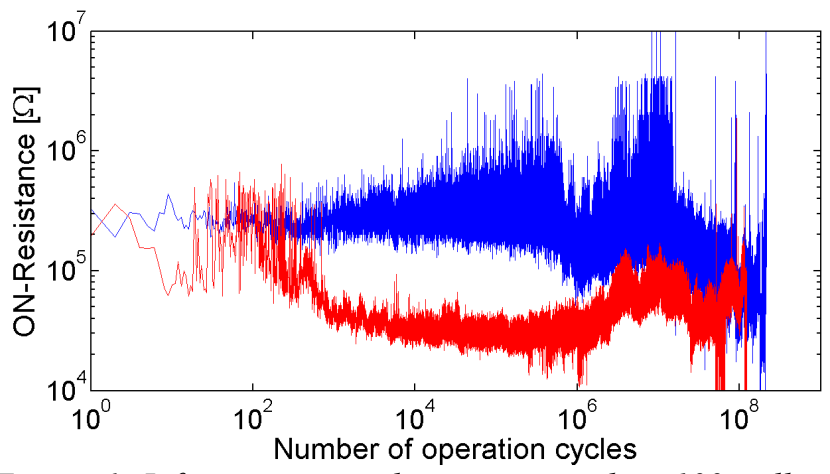

Figure 6: Lifetime test results over more than 100 million hot switching cycles with $1.6 \mathrm{~V}$ (blue) and $2.0 \mathrm{~V}$ (red). Filament formation mainly occurs in the first switching cycles (higher resistance) and for higher voltages.

Detailed analysis of the measurement data reveals additional information. $\mathrm{R}_{\mathrm{ON}}$ is often higher in the beginning of an experiment and then decreases after some time. We attribute this to the formation of multiple filaments in the contact tip, caused by the probability of the tip to contact the previously formed filament in the exact same location. Therefore, the conduction behavior becomes stable only after a number of events that seems to be linked also to the voltage drop over the contact. This is observed in the experiment run at $\mathrm{V}_{1}=2.0 \mathrm{~V}$, where the initial contact resistance is about $200 \mathrm{k} \Omega$, dropping to $<50 \mathrm{k} \Omega$ within a few hundred cycles. Closing the switches with a lower $V_{1}$ of 1.6 $\mathrm{V}$ shows a more gradual decrease of the extracted contact resistance starting from the same initial value. This is an indication that the filament formed at $1.6 \mathrm{~V}$ is either less stable or it does not contribute to the current conduction of the subsequent switching cycle as much as a filament formed at higher voltage.

\section{BUILDING BLOCK: INVERTER}

The new NEM switch proposed in this work meets all requirements for logic circuits: a reliable contact, small hysteresis, low pull-in voltage, high breakdown voltage, small size, and the capability to sustain high voltage at the output. The inverter is the classical building block to demonstrate these attributes in a single device.

Figure 7 shows an SEM micrograph of a NEMS-based inverter. The left side shows two NEM switches in an inverter configuration that drive the gate of an output switch (right side). Decoupling the inverter from the measurement setup by means of an output switch reduces the capacitive load from the measurement setup. These parasitic loads from the measurement setup are a major limitation when measuring devices with a high drain voltage as they lead to high surge currents and welding of the contact tip.

Figure 8 shows the measurement results and the measurement setup of the inverter presented in Figure 7. Figure $8 \mathrm{a}$ shows the measurement results of the NEMswitch-based inverter with $V_{\mathrm{DD}}=4 \mathrm{~V}$ and $V_{\mathrm{SS}}=-4 \mathrm{~V}$. Cycling the input signal (blue) between the two supply 
voltages results in a corresponding oscillation of the output signal $\left(V_{\text {Output }}\right)$. To limit and control the voltage on the output switch, the virtual ground of the transimpedance amplifier is set to $1.75 \mathrm{~V}$, resulting in a drain-to-source voltage drop of $2.25 \mathrm{~V}$ on the output switch.

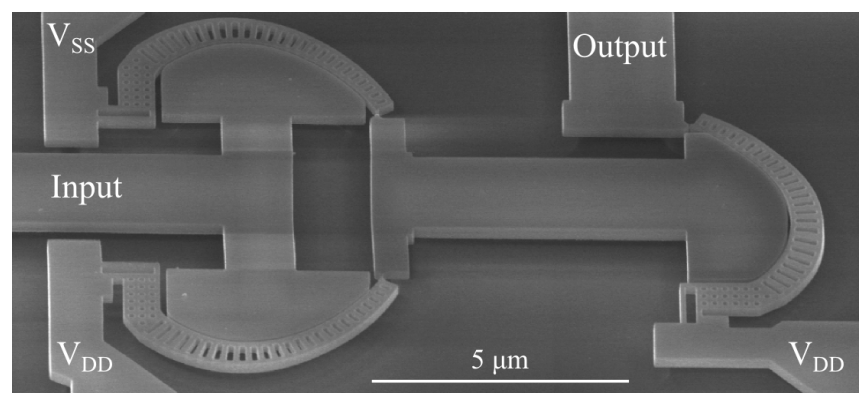

Figure 7: SEM image of an inverter driving a single output NEM switch that decouples the inverter from the measurement setup.

Figure $8 \mathrm{~b}$ shows a schematic of the measurement setup including the inverter, the output switch and the transimpedance amplifier at the output. The gain of the amplifier and the steady current in all NEM switches are limited by using discrete resistors. The measured output signal is the result of three inversions by the total setup and switches between the open state $\left(V_{\text {Output }}=1.75 \mathrm{~V}\right)$ and the closed state ( $V_{\text {Output }} \sim 0$ to $0.5 \mathrm{~V}$ ) of the output switch. However, the voltage of the internal gate must cycle through the full voltage span to create this characteristic. Therefore, we demonstrate with this inverter setup that carbon-coated NEM switches can drive additional devices over the entire supply voltage range.

(a)

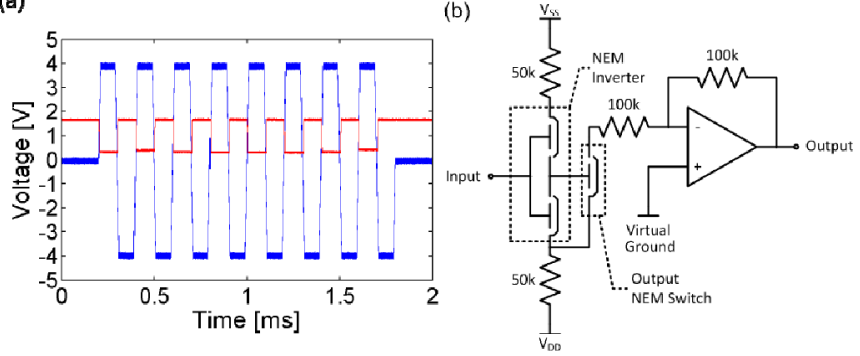

Figure 8: (a) Measurement of an inverter as shown in Figure 7, with the output connected to the virtual ground of a transimpedance amplifier (setup in (b)). The input (blue) actuates the inverter; the output (red) is measured from the inverting amplifier. The virtual ground ( $\left.V_{\text {Output }}\right)$ is set to $1.75 \mathrm{~V} .\left(V_{D D}=+4 \mathrm{~V}\right.$ and $\left.V_{S S}=-4 \mathrm{~V}\right)$.

\section{CONCLUSION}

A first evaluation of amorphous carbon (a-C) as a contact material for nanoelectromechanical (NEM) switches is presented in this paper. a-C has a low surface energy and offers an elegant way of creating nanoscale contacts by forming filaments in the a-C layer. Combining these advantages with a curved NEM switch design, we demonstrate all attributes necessary for nanomechanical logic: a reliable contact $\left(R_{\mathrm{ON}}=15 \mathrm{k} \Omega,>10^{8}\right.$ switching cycles), small hysteresis, low pull-in voltage, high breakdown voltage, small size, and the capability to sustain the supply voltage at the output. In addition, this work provides the characteristics of an inverter used as basic building block for logic circuits, revealing a way towards achieving more complex logic systems.

\section{ACKNOWLEDGEMENTS}

The authors gratefully acknowledge the support of Kevin Lister, Meinrad Tschudy, Richard Stutz and the whole team working at the IBM Binnig and Rohrer Nanotechnology Center.

The research leading to these results has received funding from the European Union's Seventh Framework Programme (FP7/2007-2011) under grant agreement No. 288670.

\section{REFERENCES}

[1] A. Knoll et al., "Fundamental scaling properties of electro-mechanical switches", New J. Phys. 14 (2012) 123007.

[2] J. Yaung et al., "Adhesive force characterization for MEM logic relays with sub-micron contacting regions", J. Microelectromech. Systems (2013), DOI: 10.1109/JMEMS.2013.2269995

[3] S.M. Kim et al., "Suspended few-layer graphene beam electromechanical switch with abrupt on-off characteristics and minimal leakage current", Appl. Phys. Lett. 99 (2011) 023103.

[4] O. Loh et al., "Carbon-Carbon Contacts for Robust Nanoelectromechanical Switches", Adv. Mater. 24 (2012) 2463.

[5] M.P. de Boer et al., "Design, fabrication, performance and reliability of Pt- and $\mathrm{RuO}_{2}$-coated microrelays tested in ultra-high purity gas environments", J. Micromech. Microeng. 22 (2012) 105027.

[6] H. Kam et al., "Design and reliability of a micro-relay technology for zero-standby-power digital logic applications", in Proc. IEEE IEDM, 2009, p. 809.

[7] A. Sebastian et al., "Resistance switching at the nanometre scale in amorphous carbon", New J. Phys. 13 (2011) 013020.

[8] D. Grogg et al., "Curved in-plane electromechanical relay for low power logic applications", J. Micromech. Microeng. 23 (2013) 025024.

\section{CONTACT}

*D. Grogg, dgr@zurich.ibm.com

*U.T. Duerig, drg@zurich.ibm.com 